\title{
101.10 Exradii of the triangle and Euler's Inequality
}

The purpose of this very short Note is to give an alternative proof of the inequality $R \geqslant 2 r$ which establishes a fundamental relation between the circumradius $R$ and the inradius $r$ of a triangle $A B C$. In our proof we use the arithmetic mean-harmonic mean inequality, which can be seen as a special case of the Cauchy-Schwarz inequality. The inequality says that for three positive numbers $x, y, z$, the arithmetic mean $\frac{1}{3}(x+y+z)$ is greater than or equal to the harmonic mean $\frac{3}{\frac{1}{x}+\frac{1}{y}+\frac{1}{z}}$. It is very natural with the circumradius and inradius to consider the exradii of the triangle $r_{a}, r_{b}, r_{c}$. As is well known, they are related by the two equations [1]

$$
\begin{gathered}
\frac{1}{r_{a}}+\frac{1}{r_{b}}+\frac{1}{r_{c}}=\frac{1}{r}, \\
r_{a}+r_{b}+r_{c}=4 R+r .
\end{gathered}
$$

What does not seem to be mentioned is that we can now apply the AM-HM inequality to these to obtain

$$
\left(r_{a}+r_{b}+r_{c}\right)\left(\frac{1}{r_{a}}+\frac{1}{r_{b}}+\frac{1}{r_{c}}\right) \geqslant 9,
$$

that is $\frac{4 R+r}{r} \geqslant 9$, which is equivalent to Euler's triangle inequality $R \geqslant 2 r$. Equality holds for $r_{a}=r_{b}=r_{c}$, which in turn implies $s-a=s-b=s-c$, and so the triangle is equilateral.

\section{Reference}

1. G. Leversha, The geometry of the triangle, UKMT (2013).

10.1017/mag.2017.18

MARTIN LUKAREVSKI

Department of Mathematics and Statistics, University 'Goce Delcev', Stip, Macedonia e-mail:martin.lukarevski@ugd.edu.mk

\subsection{An application of a morsel of Honsberger}

\section{Dedicated to the memory of Ross Honsberger}

In [1], A. Plaza proves that among all parallelograms whose diagonals have given lengths, the one with maximum perimeter is the rhombus. Plaza's short proof uses the law of cosines and the inequality between the root mean square and the arithmetic mean. This inequality says that if $x, y>0$, then

$$
\left(\frac{\sqrt{x}+\sqrt{y}}{2}\right)^{2} \leqslant \frac{x+y}{2}
$$

Feeling that this inequality is not that well-known among geometry students, I give another short proof below using (the law of cosines and) Apollonius' medians theorem. Then I show how Plaza's problem follows from one of Ross Honsberger's seemingly favourite problems. 